\title{
Existence of Bounded Solutions for Second Order Dynamic Equations
}

\author{
ELVAN AKIN ${ }^{a, b}$, MARTIN BOHNER ${ }^{a, b}$, LYNN ERBE $^{a, b}$ and \\ ALLAN PETERSON $\mathrm{N}^{\mathrm{a}, \mathrm{b}, *}$
}

Department of Mathematics and Statistics, University of Nebraska-Lincoln, Lincoln, XP 68588-0323, USA; Department of Mathematics and Statistics, University of Missouri-Rolla. 115, Rolla Building, MO 65409-0020, USA

(Received 19 September 2001; Revised 19 September 2001; In final form 19 September 2001)

This Paper is dedicated to Professor Lynn H. Erbe

In this paper, we will give sufficient conditions for a nonhomogeneous dynamic self-adjoint equation on a time scale to have a zero tending solution. We also give sufficient conditions that guarantees that for each constant $C$ there is a unique bounded solution on $[a, \infty)$ with $y(a)=C$.

Keywords: Measure chains; Time scales; Self-adjoint; Dynamic equations

\section{INTRODUCTION}

For completeness, we introduce the following concepts related to the notion of time scales. We say $\mathbb{T}$ is a time scale, provided, it is a closed subset of the

*Corresponding author. Address: Department of Mathematics, University of NebraskaLincoln, Lincoln, NE 68588-0323, USA. E-mail: apeterso@math.unl.edu.

ISSN 1023-6198 @ 2001 Taylor \& Francis Ltd DOI: $10.1080 / 10236190290017414$ 
real numbers $\mathbb{R}$. We assume throughout that $\mathbb{T}$ has the topology that it inherits from the standard topology on the real numbers $\mathbb{R}$. We also assume throughout this paper that $a \in \mathbb{T}$ and $\sup \mathbb{T}=\infty$.

DEFINITION 1 We define the forward jump operator $\sigma$, for $t \in \mathbb{T}$, by

$$
\sigma(t):=\inf \{\tau>t: \tau \in \mathbb{T}\}
$$

and the backward jump operator $\rho$, for $t \in \mathbb{T}, t>\inf \mathbb{T}$, by

$$
\rho(t):=\sup \{\tau<t: \tau \in \mathbb{T}\} .
$$

If $\sigma(t)>t$, we say $t$ is right-scattered, while if $\rho(t)<t$ we say $t$ is leftscattered. If $\sigma(t)=t$ we say $t$ is right-dense, while if $\rho(t)=t$ we say $t$ is left-dense. A function $f: \mathbb{T} \rightarrow \mathbb{R}$ is said to be right-dense continuous provided $f$ is continuous at right-dense points in $T$ and at left-dense points in $\mathbb{T}$, left hand limits exist and are finite. We shall also use the notation $\mu(t): \sigma(t)-t$ and we call $\mu$ the graininess function. Finally, if $f: \mathbb{T} \rightarrow \mathbb{R}$ is a function, then we define the function $f^{\sigma}: \mathbb{T} \rightarrow \mathbb{R}$ by

$$
f^{\sigma}(t)=f(\sigma(t)) \text { for all } t \in \mathbb{T} .
$$

i.e. $f^{\sigma}=f \circ \sigma$. Similarly, $f^{\rho}=f^{\circ} \rho$.

DeFINITION 2 We define the interval in $\mathbb{T}$

$$
[a, \infty):=\{t \in \mathbb{T} \text { such that } t \geq a\} .
$$

The notion of a measure chain was introduced by Hilger [10]. Related work on the calculus of measure chains may be found in Refs. [2,3,7-9]. For an introduction to dynamic equations on time scales see Refs. $[1,5,6,11]$.

Defintion 3 Assume $x: \mathbb{T} \rightarrow \mathbb{R}$ and fix $t \in \mathbb{T}$, then we define $x^{\Delta}(t)$ to be the number (provided it exists) with the property that given any $\epsilon>0$, there is a neighborhood $U$ of $t$ such that

$$
\| x(\sigma(t))-x(s)\rfloor-x^{\Delta}(t)\lfloor\sigma(t)-s \| \leq \epsilon|\sigma(t)-s|,
$$

for all $s \in U$. We call $x^{\Delta}(t)$ the delta derivative of $x(t)$ at $t$. 
It can be shown that if $x: \mathbb{T} \rightarrow \mathbb{R}$ is continuous at $t \in \mathbb{T}$ and $t$ is rightscattered, then

$$
x^{\Delta}(t)=\frac{x(\sigma(t))-x(t)}{\sigma(t)-t} .
$$

Note that if $\mathbb{T}=\mathbb{Z}$, the integers, then

$$
x^{\Delta}(t)=\Delta x(t):=x(t+1)-x(t) .
$$

If $t$ is right-dense, then

$$
x^{\Lambda}(t)=\lim _{s \rightarrow t} \frac{x(t)-x(s)}{t-s}
$$

if the limit exists. In particular, if $\mathbb{T}=\mathbb{R}$, the real numbers, then $x^{\Delta}(t)=$ $x^{\prime}(t)$.

Also an integral $\int_{a}^{b} h(t) \Delta t$ can be defined (see Ref. [6]). It turns out if $\mathbb{T}=\mathbb{R}$, then

$$
\int_{a}^{b} h(t) \Delta t=\int_{a}^{b} h(t) \mathrm{d} t
$$

is the Riemann integral and if $\mathbb{T}=\mathbb{Z}$ and $a<b$ are integers, then

$$
\int_{a}^{b} h(t) \Delta t=\sum_{t=a}^{b-1} h(t)
$$

\section{MAIN RESULTS}

In this section, we will state and prove out main results. We will mainly be concerned with the linear nonhomogeneous dynamic equation with variable coefficients in self-adjoint form:

$$
\left(p(t) y^{\Delta}(t)\right)^{\Delta}+q(t) y^{\sigma}(t)=f(t)
$$

where $p, q$, and $f$ are rd-continuous functions on $\mathbb{T}$ and $p$ is a positive function.

The following result guarantees the existence of a solution of Eq. (1) converging to zero as $t \rightarrow \infty$ independent of whether it is oscillatory or nonoscillatory. 


\section{THEOREM 4 If}

$$
\begin{aligned}
& \text { (i) } p(t)>0, \quad q(t) \geq 0 \text { for all } t \in[a, \infty), \\
& \text { (ii) } \int_{a}^{\infty} \frac{1}{p(\tau)} \Delta \tau<\infty, \\
& \text { (iii) } \int_{a}^{\infty} q(\tau) P^{\sigma}(\tau) \Delta \tau<\infty, \quad \text { where } P(t):=\int_{t}^{\infty} \frac{1}{p(s)} \Delta s, \\
& \text { (iv) } \int_{a}^{\infty} f(\tau) \Delta \tau<\infty,
\end{aligned}
$$

then Eq. (1) has a solution which converges to zero as $t \rightarrow \infty$.

Proof Let

$$
F(t):=\int_{t}^{\infty} f(\tau) \Delta \tau
$$

and

$$
K(t):=\int_{t}^{\infty} \frac{F(s)}{p(s)} \Delta s
$$

where $K$ is well defined follows from (ii) and (iv). Therefore $K(t) \rightarrow 0$ as $t \rightarrow \infty$. Also, $P(t) \rightarrow 0$ as $t \rightarrow \infty$ and $\int_{t}^{\infty} q(\tau) P^{\sigma}(\tau) \Delta \tau \rightarrow 0$ as $t \rightarrow \infty$ because of (ii) and (iii), respectively.

By (iii) choose $T \in[a, \infty)$ sufficiently large so that

$$
\alpha:=\int_{T}^{\infty} q(\tau) P^{\sigma}(\tau) \Delta \tau \in(0,1)
$$

Let $X$ be the Banach Space of all continuous functions $x:[T, \infty) \mapsto \mathbb{R}$ which converge to zero with the norm $\|\cdot\|$ defined by

$$
\|y\|=\sup \{|y(t)|: t \in[T, \infty)\},
$$

and define the operator $A$ on $X$ by

$$
A y(t):=K(t)+L y(t), \quad y \in X,
$$

where $K$ is defined by Eq. (2) and $L$ is the operator defined by

$$
L y(t):=P(t) \int_{\tau}^{t} q(\tau) y^{\sigma}(\tau) \Delta \tau+\int_{t}^{\infty} q(\tau) P^{\sigma}(\tau) y^{\sigma}(\tau) \Delta \tau
$$


for all $t \in[T, \infty)$. It is clear that $A y$ is continuous on $[T, \infty)$. Hence to show $A: X \mapsto X$ it remains to show that $\lim _{t \rightarrow \infty} A y(t)=0$. Since $K(t) \rightarrow 0$ as $t \rightarrow \infty$, we need only to show that $L y(t) \rightarrow 0$ as $t \rightarrow \infty$. To show this, it suffices to show that if

$$
x(t):=P(t) \int_{T}^{t} q(\tau) y^{\sigma}(\tau) \Delta \tau
$$

then $\lim _{t \rightarrow \infty} x(t)=0$. To see this, let $\varepsilon>0$ be given. Choose $t_{0} \in \mathbb{T}$ such that $t_{0} \geq T$ and

$$
\left|y^{\sigma}(t)\right|<\varepsilon \quad \text { for all } t>t_{0}
$$

For this $t_{0}$, set

$$
\beta=\left|\int_{T}^{t_{0}} q(\tau) y^{\sigma}(\tau) \Delta \tau\right|
$$

and since $P(t) \rightarrow 0$ as $t \rightarrow \infty$ we can choose $t_{1} \in\left[t_{0}, \infty\right)$ such that

$$
P(t) \beta<\varepsilon
$$

for all $t \geq t_{1}$. Then for all $t \geq t_{1}$,

$$
\begin{aligned}
|x(t)| & =\left|P(t) \int_{T}^{t} q(\tau) y^{\sigma}(\tau) \Delta \tau\right|=\left|P(t) \int_{T}^{t_{0}} q(\tau) y^{\sigma}(\tau) \Delta \tau+P(t) \int_{t_{0}}^{t} q(\tau) y^{\sigma}(\tau) \Delta \tau\right| \\
& \leq P(t)\left|\int_{T}^{t_{0}} q(\tau) y^{\sigma}(\tau) \Delta \tau\right|+P(t) \int_{t_{0}}^{t} q(\tau)\left|y^{\sigma}(\tau)\right| \Delta \tau \\
& =P(t) \beta+\int_{t_{0}}^{t} q(\tau) P^{\sigma}(\tau)\left|y^{\sigma}(\tau)\right| \Delta \tau<\varepsilon+\varepsilon \int_{t_{0}}^{t} q(\tau) P^{\sigma}(\tau) \Delta \tau \\
& <\varepsilon(1+\alpha)
\end{aligned}
$$

since $P$ is decreasing and we used Eqs. (7), (8), (6), and (3), respectively, (here we used $P(t) \int_{t_{0}}^{t} q(\tau) \Delta \tau<\int_{t_{0}}^{t} q(\tau) P^{\sigma}(\tau) \Delta \tau$ but this is easy to verify). Hence $\lim _{t \rightarrow \infty} x(t)=0$ and so $A: X \mapsto X$. Next we show that $A$ is a contraction 
mapping on $X$. Let $y, z \in X, t \geq T$ and consider

$$
\begin{aligned}
& |A y(t)-A z(t)|=|L y(t)-L z(t)| \leq P(t) \int_{T}^{t} q(\tau)\left|y^{\sigma}(\tau)-z^{\sigma}(\tau)\right| \Delta \tau \\
& \quad+\int_{t}^{\infty} q(\tau) P^{\sigma}(\tau)\left|y^{\sigma}(\tau)-z^{\sigma}(\tau)\right| \Delta \tau \\
& \quad \leq \int_{T}^{t} q(\tau) P^{\sigma}(\tau)\left|y^{\sigma}(\tau)-z^{\sigma}(\tau)\right| \Delta \tau+\int_{t}^{\infty} q(\tau) P^{\sigma}(\tau)\left|y^{\sigma}(\tau)-z^{\sigma}(\tau)\right| \Delta \tau \\
& =\int_{t}^{\infty} q(\tau) P^{\sigma}(\tau)\left|y^{\sigma}(\tau)-z^{\sigma}(\tau)\right| \Delta \tau \leq\|y-z\| \int_{t}^{\infty} q(\tau) P^{\sigma}(\tau) \Delta \tau=\alpha\|y-z\|
\end{aligned}
$$

since $P$ is decreasing and by Eq. (3). Therefore

$$
\|A y-A z\| \leq \alpha\|y-z\|
$$

for all $y, z \in X$ and so $A$ is a contraction mapping on $X$. Hence by the Banach Fixed Point Theorem, $A$ has a unique fixed point $y \in X$. Since $y=A y$, we have $y(t)$ converges to 0 as $t \rightarrow \infty$. It remains to show that Eq. (9) is a solution of Eq. (1). Since $y=A y$,

$$
y(t)=K(t)+P(t) \int_{T}^{t} q(\tau) y^{\sigma}(\tau) \Delta \tau+\int_{t}^{\infty} q(\tau) P^{\sigma}(\tau) y^{\sigma}(\tau) \Delta \tau
$$

for all $t \geq T$. Taking the derivative of both sides we get

$$
\begin{gathered}
y^{\Delta}(t)=K^{\Delta}(t)+P^{\sigma}(t) q(t) y^{\sigma}(t)+P^{\Delta}(t) \int_{T}^{t} q(\tau) y^{\sigma}(\tau) \Delta \tau+q(t) P^{\sigma}(t) y^{\sigma}(t) \\
y^{\Delta}(t)=-\frac{F(t)}{p(t)}-\frac{1}{p(t)} \int_{T}^{t} q(\tau) y^{\sigma}(\tau) \Delta \tau
\end{gathered}
$$

and so

$$
p(t) y^{\Delta}(t)=-F(t)-\int_{T}^{t} q(\tau) y^{\sigma}(\tau) \Delta \tau
$$

and therefore

$$
\left(p(t) y^{\Delta}(t)\right)^{\Delta}=f(t)-q(t) y^{\sigma}(t) .
$$

So we get the desired result. 
DEFINITION 5 A function $x$ is called eventually positive (eventually negative) provided there is a $T \in[a, \infty)$ such that $x(t) \geq 0(x(t) \leq 0)$ for all $t \in[T, \infty)$.

COROLLARY 6 Assume that (i)-(iv) of Theorem 4 hold. If $f$ is eventually positive (eventually negative), then Eq. (1) has an eventually positive (eventually negative) solution converging to zero as $t \rightarrow \infty$.

Proof Assume $f$ is eventually positive. Without loss of generality we can assume that $f(t) \geq 0$ for all $t \in[T, \infty)$, where $T$ is as in the proof of Theorem 4. Let $A$ be defined by Eqs. (4) and (5) and consider the closed subset $S^{+} \subset X$ defined by

$$
S^{+}=\{y \in X: y(t) \geq 0 \quad \text { for all } t \in[T, \infty)\} .
$$

As $K(t) \geq 0$ for all $t \in[T, \infty), A S^{+} \subset S^{+}$. By the same argument as in the proof of Theorem 1, one can show that $A$ is a contraction on $S^{+}$. Therefore we get the desired result in the eventual positive case. The eventual negative case is similar.

REMARK 7 Since $(1 / t)^{\Delta}=-(1 / t \sigma(t)), \int_{1}^{\infty}(1 / t \sigma(t)) \Delta t=1$.

Example 8 Assume sup $\mathbb{T}=\infty$. Consider the dynamic equation

$$
\left(t \sigma(t) x^{\Delta}(t)\right)^{\Delta}+\frac{1}{t \sigma(t)} x^{\sigma}(t)-\frac{1}{t(\sigma(t))^{2}}
$$

on $[1, \infty) \cap \mathrm{T}$. Using Remark 7 we see that the hypotheses of Theorem 1 are satisfied. One of its solutions (use Remark 7) on $\rceil$ is $x(t)=1 / t$ which converges to zero as $t \rightarrow \infty$.

We now consider the analogue of Theorem 4 for the self-adjoint equation

$$
\left[p(t) y^{\Delta}(t)\right]^{\nabla}+q(t) y(t)=f(t)
$$

which was introduced by Atici and Guseinov [5]. When considering this equation we assume that $f: \mathbb{T} \rightarrow \mathbb{R}$ is continuous, $p: \mathbb{T} \rightarrow \mathbb{R}$ is continuous and positive, and $q: \mathbb{T} \rightarrow \mathbb{R}$ is continuous. For the definition of the nabla derivative $\nabla$ and the nabla integral $\int_{a}^{b} h(t) \nabla t$ see Refs. $[4,6]$. 
THEOREM 9 If

(i) $p(t)>0, q(t) \geq 0$ for all $t \in[a, \infty)$,

(ii) $\int_{a}^{\infty} \frac{1}{p(\tau)} \nabla \tau<\infty, \quad \int_{a}^{\infty} \frac{1}{p(\tau)} \Delta \tau<\infty$,

(iii) $\int_{a}^{\infty} q^{\sigma}(\tau) P^{\sigma}(\tau) \Delta \tau<\infty$, where $P(t):=\int_{t}^{\infty} \frac{1}{p(s)} \nabla s$,

(iv) $\int_{a}^{\infty} f(\tau) \nabla \tau<\infty$

then Eq. (1) has a solution which converges to zero as $t \rightarrow \infty$.

Proof The proof is very similar to the proof of Theorem 4 so we will just indicate how we define some things differently for this case and do the last part of the proof. In this proof $F, P$, and $K$ are defined by

$$
F(t):=\int_{t}^{\infty} f(s) \nabla s, \quad P(t):=\int_{t}^{\infty} \frac{1}{p(s)} \nabla s, \quad K(t):=\int_{t}^{\infty} \frac{F(s)}{p(s)} \Delta s
$$

and $L$ and $A$ are defined by

$$
L y(t):=P(t) \int_{T}^{t} q(\tau) y(\tau) \nabla \tau+\int_{t}^{\infty} P^{\sigma}(\tau) q^{\sigma}(\tau) y^{\sigma}(\tau) \Delta \tau
$$

and

$$
A y(t):=L y(t)+K(t),
$$

respectively, where $T$ is sufficiently large. We just show that if $y$ is a fixed point of $A$, then $y$ is a solution of Eq. (11). To see this consider

$$
y(t)=A y(t)=P(t) \int_{T}^{t} q(\tau) y(\tau) \nabla \tau+\int_{t}^{\infty} P^{\sigma}(\tau) q^{\sigma}(\tau) y^{\sigma}(\tau) \Delta \tau+K(t)
$$

Taking the delta derivative of both sides and using the formula (see Ref. [4] or [6])

$$
\left(\int_{a}^{t} h(s) \nabla s\right)^{\Delta}=h(\sigma(t))
$$


we get

$$
\begin{aligned}
y^{\Delta}(t)= & P^{\sigma}(t) q^{\sigma}(t) y^{\sigma}(t)-\frac{1}{p(t)} \int_{T}^{t} q(\tau) y(\tau) \nabla \tau-P^{\sigma}(t) q^{\sigma}(t) y^{\sigma}(t) \\
& -\frac{1}{p(t)} \int_{t}^{\infty} f(\tau) \nabla \tau .
\end{aligned}
$$

It follows that

$$
p(t) y^{\Delta}(t)=-\int_{T}^{t} q(\tau) y(\tau) \nabla \tau-\int_{T}^{\infty} f(\tau) \nabla \tau
$$

Taking the nabla derivative of both sides we get the desired result

$$
\left[p(t) y^{\Delta}(t)\right]^{\nabla}=-q(t) y(t)+f(t) .
$$

In the next result we relax the positivity of $q$ and the condition on $f$ guaranteeing the existence of bounded solutions for Eq. (1).

THEOREM 10 Assume there are constants $\mu, \bar{\mu}$ such that $0<\underline{\mu} \leq \mu(t) \leq$ $\bar{\mu}$ for all $t \in[a, \infty)$.

If the conditions

$$
\text { (i) } 0<\alpha \leq p(t) \leq \beta, \text { for all } t \in[a, \infty) \text {, }
$$

(ii) either

$$
q(t) \leq \gamma<0, \quad \text { for all } t \in[a, \infty),
$$

or there is a constant $\delta>2$ such that

$$
\frac{q(t) \mu^{2}(t) \mu^{\sigma}(t)}{p^{\sigma}(t) \mu(t)+p(t) \mu^{\sigma}(t)} \geq \delta>2 \text { for all } t \in[a, \infty),
$$

(iii) $f$ is bounded on $[a, \infty)$, hold, then for each number $C$ there is a unique bounded solution of Eq. (1) with $y(a)=C$.

Proof Equation (1) can be written in the form:

$$
\begin{aligned}
& p^{\sigma}(t) \mu(t) y^{\sigma^{2}}(t)-\left[p^{\sigma}(t) \mu(t)+p(t) \mu^{\sigma}(t)-q(t) \mu^{2}(t) \mu^{\sigma}(t)\right] y^{\sigma}(t) \\
& \quad+p(t) \mu^{\sigma}(t) y(t)=\mu^{2}(t) \mu^{\sigma}(t) f(t)
\end{aligned}
$$

hence

$$
y^{\sigma}(t)=\frac{p^{\sigma}(t) \mu(t) y^{\sigma^{2}}(t)+p(t) \mu^{\sigma}(t) y(t)-\mu^{2}(t) \mu^{\sigma}(t) f(t)}{p^{\sigma}(t) \mu(t)+p(t) \mu^{\sigma}(t)-q(t) \mu^{2}(t) \mu^{\sigma}(t)}
$$


Let $t=\rho(s)$, then

$$
\begin{aligned}
& y(s)=\frac{p(s) \mu^{\rho}(s) y^{\sigma}(s)+p^{\rho}(s) \mu(s) y^{\rho}(s)-\mu^{2}(\rho(s)) \mu(s) f^{\rho}(s)}{p(s) \mu^{\rho}(s)+p^{\rho}(s) \mu(s)-q^{\rho}(s) \mu^{2}(\rho(s)) \mu(s)}, \\
& \quad \text { for } s \in[\sigma(a), \infty) .
\end{aligned}
$$

Consider the Banach Space $X$ of bounded functions on $[a, \infty)$ with the norm $\|y\|=\sup |y(t)| t \in[a, \infty)$. Define the operator $T$ by

$$
\begin{aligned}
& T y(a)=C, \\
& T y(s)=\frac{p(s) \mu^{\rho}(s) y^{\sigma}(s)+p^{\rho}(s) \mu(s) y^{\rho}(s)-\mu^{2}(\rho(s)) \mu(s) f^{\rho}(s)}{p(s) \mu^{\rho}(s)+p^{\rho}(s) \mu(s)-q^{\rho}(s) \mu^{2}(\rho(s)) \mu(s)}, \\
& \quad \text { for all } s \geq \sigma(a) .
\end{aligned}
$$

It is clear that $T: X \mapsto X$. Since the numerator is bounded above and the denominator is bounded away from zero, $T$ is bounded.

Consider the case when condition (12) is satisfied. In this case $p(s) \mu^{\rho}(s)+p^{\rho}(s) \mu(s)-q^{\rho}(s) \mu^{2}(\rho(s)) \mu(s)>0$. We now show that $T$ is a contraction mapping on $X$. Let $y, z \in X$, and $s \geq \sigma(a)$. Then

$$
\begin{aligned}
|T y(s)-T z(s)| & =\left|\frac{p(s) \mu^{\rho}(s)\left(y^{\sigma}(s)-z^{\sigma}(s)\right)+p^{\rho}(s) \mu(s)\left(y^{\rho}(s)-z^{\rho}(s)\right)}{p(s) \mu^{\rho}(s)+p^{\rho}(s) \mu(s)-q^{\rho}(s) \mu^{2}(\rho(s)) \mu(s)}\right| \\
& \leq \frac{p(s) \mu^{\rho}(s)\left|y^{\sigma}(s)-z^{\sigma}(s)\right|-p^{\rho}(s) \mu(s)\left|y^{\rho}(s)-? ? z^{\rho}(s)\right|}{p(s) \mu^{\rho}(s)+p^{\rho}(s) \mu(s)-q^{\rho}(s) \mu^{2}(\rho(s)) \mu(s)} \\
& \leq \frac{p(s) \mu^{\rho}(s)+p^{\rho}(s) \mu(s)}{p(s) \mu^{\rho}(s)+p^{\rho}(s) \mu(s)-q^{\rho}(s) \mu^{2}(\rho(s)) \mu(s)}\|y-z\|
\end{aligned}
$$

for $s \in[\sigma(a), \infty)$. Consider

$$
\begin{gathered}
\frac{p(s) \mu^{\rho}(s)+p^{\rho}(s) \mu(s)}{p(s) \mu^{\rho}(s)+p^{\rho}(s) \mu(s)-q^{\rho}(s) \mu^{2}(\rho(s)) \mu(s)} \\
=\frac{1}{1-\left(\frac{q^{\rho}(s) \mu^{2}(\rho(s)) \mu(s)}{p(s) \mu^{\rho}(s)+p^{\rho}(s) \mu(s)}\right)} \cdot
\end{gathered}
$$

But from (i), Eq. (12) and the assumptions on $\mu(t)$, we have

$$
\frac{q^{\rho}(s) \mu^{2}(\rho(s)) \mu(s)}{p(s) \mu^{\rho}(s)+p^{\rho}(s) \mu(s)} \leq \frac{\gamma \mu^{3}}{2 \beta \bar{\mu}}:=\kappa<0
$$


for $s \in[\sigma(a), \infty)$. Therefore

$$
\begin{aligned}
|T y(s)-T z(s)| & \leq \frac{p(s) \mu^{\rho}(s)+p^{\rho}(s) \mu(s)}{p(s) \mu^{\rho}(s)+p^{\rho}(s) \mu(s)-q^{\rho}(s) \mu^{2}(\rho(s)) \mu(s)} \\
& \leq \frac{1}{1-\kappa}<1
\end{aligned}
$$

for all $s \in[T y(a), \infty)$. If $s=a$, then

$$
|T y(a)-T z(a)|=0 \leq \frac{1}{1-\kappa}\|y-z\| .
$$

Therefore

$$
|T y(s)-T z(s)|<\frac{1}{1-\kappa}\|y-z\|, \quad \text { for all } s \in[a, \infty) .
$$

This implies that

$$
\|T y-T z\| \leq \frac{1}{1-\kappa}\|y-z\|
$$

and so $T$ is a contraction mapping on $X$. Hence by the Banach Fixed Point Theorem, $T$ has a unique fixed point $y$. It follows that

$$
\begin{aligned}
& y(a)=T y(a)=C, \\
& y(s)=T y(s)=\frac{p(s) \mu^{\rho}(s) y^{\sigma}(s)+p^{\rho}(s) \mu(s) y^{\rho}(s)-\mu^{2}(\rho(s)) \mu(s) f^{\rho}(s)}{p(s) \mu^{\rho}(s)+p^{\rho}(s) \mu(s)-q^{\rho}(s) \mu^{2}(\rho(s)) \mu(s)}, \\
& \quad \text { for all } s \geq \sigma(a)
\end{aligned}
$$

is the unique bounded solution of Eq. (1) satisfying $y(a)=C$.

Finally consider the case when Eq. (13) is satisfied. Again we will show that $T$ is a contraction mapping on $X$. Let $y, z \in X$, and $s \geq \sigma(a)$. Then

$$
\begin{aligned}
|T y(s)-T z(s)| & =\left|\frac{p(s) \mu^{\rho}(s)\left(y^{\sigma}(s)-z^{\sigma}(s)\right)+p^{\rho}(s) \mu(s)\left(y^{\rho}(s)-z^{\rho}(s)\right)}{p(s) \mu^{\rho}(s)+p^{\rho}(s) \mu(s)-q^{\rho}(s) \mu^{2}(\rho(s)) \mu(s)}\right| \\
& \leq \frac{p(s) \mu^{\rho}(s)\left|y^{\sigma}(s)-z^{\sigma}(s)\right|+p^{\rho}(s) \mu(s)\left|y^{\rho}(s)-z^{\rho}(s)\right|}{\left|p(s) \mu^{\rho}(s)+p^{\rho}(s) \mu(s)-q^{\rho}(s) \mu^{2}(\rho(s)) \mu(s)\right|} \\
& \leq \frac{p(s) \mu^{\rho}(s)+p^{\rho}(s) \mu(s)}{\left|p(s) \mu^{\rho}(s)+p^{\rho}(s) \mu(s)-q^{\rho}(s) \mu^{2}(\rho(s)) \mu(s)\right|}\|y-z\|
\end{aligned}
$$


on $[\sigma(a), \infty)$. Consider

$$
\begin{gathered}
\frac{p(s) \mu^{\rho}(s)+p^{\rho}(s) \mu(s)}{p(s) \mu^{\rho}(s) \mid p^{\rho}(s) \mu(s)-q^{\rho}(s) \mu^{2}(\rho(s)) \mu(s)} \\
=\frac{1}{1-\left(\frac{q^{\rho}(s) \mu^{2}(\rho(s)) \mu(s)}{p(s) \mu^{\rho}(s)+p^{\rho}(s) \mu(s)}\right)} .
\end{gathered}
$$

But from (ii), Eq. (13) we have

$$
2<\delta \leq \frac{q^{\rho}(s) \mu^{2}(\rho(s)) \mu(s)}{p(s) \mu^{\rho}(s)+p^{\rho}(s) \mu(s)}
$$

for $s \in[\sigma(a), \infty)$. This implies that

$$
\frac{1}{1-\delta} \leq \frac{1}{1-\left(\frac{q^{\rho}(s) \mu^{2}(\rho(s)) \mu(s)}{p(s) \mu^{\rho}(s)+p^{\rho}(s) \mu(s)}\right)}<0
$$

for $s \in[\sigma(a), \infty)$. Therefore

$$
|T y(s)-T z(s)| \leq\left|\frac{p(s) \mu^{\rho}(s) \mid p^{\rho}(s) \mu(s)}{p(s) \mu^{\rho}(s) \mid p^{p}(s) \mu(s)-q^{\rho}(s) \mu^{2}(\rho(s)) \mu(s)}\right| \leq \frac{1}{\delta-1}<1
$$

for all $s \in[\sigma(a), \infty)$. If $s=a$, then

$$
|T y(a)-T z(a)|=0 \leq \frac{1}{\delta-1}\|y-z\| .
$$

Therefore

$$
|T y(s)-T z(s)| \leq \frac{1}{\delta-1}\|y-z\|, \quad \text { for all } s \in[a, \infty) .
$$

Hence

$$
\|T y-T z\| \leq \frac{1}{\delta-1}\|y-z\|
$$

and so $T$ is a contraction mapping on $X$. By the Banach Fixed Point Theorem, $T$ has a unique fixed point $y$. It follows that

$$
y(a)=T y(a)=C,
$$




$$
y(s)=T y(s)=\frac{p(s) \mu^{\rho}(s) y^{\sigma}(s)+p^{\rho}(s) \mu(s) y^{\rho}(s)-\mu^{2}(\rho(s)) \mu(s) f^{\rho}(s)}{p(s) \mu^{\rho}(s)+p^{\rho}(s) \mu(s)-q^{\rho}(s) \mu^{2}(\rho(s)) \mu(s)}
$$

for all $s \geq \sigma(a)$

is the unique bounded solution of Eq. (1) satisfying $y(a)=C$.

\section{Acknowledgements}

Research was supported by NSF Grant 0072505.

\section{References}

[1] Agarwal, R. and Bohner, M. (1999) "Basic calculus on time scales and some of its applications", Results Math. 35, 3-22.

[2] Agarwal, R. and Boher, M. (1998) "Quadratic functionals for second order matrix equations on time scales", Non-linear Anal. 33, 675-692.

[3] Agarwal, R., Bohner, M. and Wong, P. (1999) "Sturm-Liouville eigenvalue problems on time scales", Appl. Math. Comput. 99, 153-166.

[4] Atici, F.M. and Guseinov, G.Sh. (2001) "On Green's functions and positive solutions for boundary value problems on time scales", J. Comput. Appl. Math., Special Issue on "Dynamic Equations on Time Scales", edited by R.P. Agarwal, M. Bohner, and D.O'Regan, to appear.

[5] Aulbach, B. and Hilger, S. (1990) "Linear dynamic processes with inhomogeneous time scale", Nonlinear Dynamics and Quantum Dynamical Systems (Gaussig, 1990) Math. Res., (Akademie Verlag, Berlin) Vol. 59, pp 9-20.

[6] Bohner, M. and Peterson, A. (2001) Dynamic Equation on Time Scales: An Introduction with Applications (Birkhäuser, Boston).

[7] Erbe, L. and Hilger, S. (1993) "Sturmian theory on measure chains", Diff. Eqs Dynam. Syst. 1(3), 223-244.

[8] Erbe, L.H. and Peterson, A. (1999) "Green's functions and comparison theorems for differential equations on measure chains", Dynam. Contin. Discrete Impuls. Syst. 6, $137-212$.

[9] Erbe, L. and Peterson, A. (2001) "Oscillation criteria for second order matrix dynamic equations on a time scale", J. Comput. Appl. Math., Special Issue on Dynamic Equations on Time Scales, edited by R.P. Agarwal, M. Bohner, and D. O'Regan, to appear.

[10] Hilger, S. (1990) "Analysis on measure chains-a unified approach to continuous and discrete calculus", Results Math. 18, 18-56.

[11] Kaymakcalan, B., Lakshmikantham, V. and Sivasundaram, S. (1996) Dynamic Systems on Measure Chains Mathematics and its Applications, (Kluwer Academic Publishers, Dordrecht) Vol. 370. 